\title{
Mid-Level Management Style in Healthcare
}

\author{
Jamion Lewis ${ }^{1}$, Kruti Lehenbauer ${ }^{2}$ \\ ${ }^{I} D B A$ Student, University of the IncarnateWord, San Antonio, USA,jslewis1@student.uiwtx.edu \\ ${ }^{2}$ Professor of Business, University of the Incarnate Word, San Antonio, USA, Lehenbau@uiwtx.edu
}

\begin{abstract}
The purpose of this paper is to recommend a quantitative model based on the outcomes from a comprehensive literature review of multiple studies to identify the most effective leadership style among midlevel managers in a healthcare setting. This literature review includes theories of leadership and discusses the usual leadership methods associated with mid-level managers. This study addresses the effectiveness of transactional, transformational, and passive leadership styles of middle level managers in healthcare organizations. The proposed quantitative non-experimental correlational study would examine the statistical relationships between the independent variables of three leadership styles (transformational, transactional, and passive leadership) and the dependent variable of leadership effectiveness among mid-level managers in the healthcare industry. This literature analysis proposes how to clearly define the variables transformational leadership, transactional leadership, passive leadership, and leader effectiveness which would eventually make it possible to quantify and measure them.
\end{abstract}

KEYWORDS: Middle Management, Leadership, Healthcare, Quantitative Model

\section{Introduction}

The Healthcare Industry in the United States is continuing to present challenges in terms of both quality and efficiency (Pelote \& Route 2007). According to Lantz \& Maryland (2008), the healthcare industry has created over \$1 trillion in avoidable costs due to inefficiencies. Aitken (2013) discussed that considerable research on healthcare system inefficiencies in the U.S. has been accomplished based on the urgency of cost containment and that the results have yielded a wide range of significant values. According to IMS Institute for Healthcare Informatics estimates, healthcare costs caused by improper and unnecessary use of medicines exceeded \$200 billion in 2012 which accounted for $8 \%$ of the national healthcare spending that year (Aitken 2013). Healthcare leaders have the ability to influence subordinates to engage in prime performance to mitigate inefficiency when faced with challenges. Chilgren (2008) stated that healthcare mid-level managers are one of the best instruments for success in terms of quality in healthcare organizations, as they can directly impact change and develop quality processes toward patient expectations by clearly identify outcomes and empower employees to achieve those goals (Chilgren 2008). Managers can expect a considerable return on investment with a successful quality program, along with satisfied patients and staff and improved clinical outcomes (Chilgren 2008). Leadership impacts communication, the effectiveness of the work environment, and the organization ability to accomplish goals. Strong leadership is needed in the healthcare environment, and the absence of it is a paramount issue (Lantz \& Maryland 2008).

This paper will examine the relationships between the leadership style and its effectiveness among middle management in the healthcare environment by conducting an extensive literature review of existing studies. This qualitative study will address the problem of the shortfall in leadership competencies among healthcare mid-level managers that have resulted in inefficiencies and subpar quality healthcare (North 2008). Mid-level managers in healthcare organizations must be prepared to appropriately handle challenges related to leadership competencies, as appropriate leadership is vital to a healthcare systems survival (Spinelli 2006). McAlearney (2008) states that driving efficiency in all aspects of a healthcare organization is critical to reducing expenses and decreasing spending. Effective management in a healthcare organization that influences others to perform efficiently is in an imperative aspect in the changing environment of the healthcare industry (Wurster 2009).

The goal of this paper is to seek out literature that examines the relationships between the independent variables of three leadership styles (transformational, transactional, and passive leadership) and the dependent variable of leadership effectiveness among mid-level managers, with specific focus on the healthcare industry. We predict that the results from this literature analysis would be applicable to any 
healthcare organization. To this effect, we focused primarily on literature that addressed one or more of the following research questions:

1. What is the relationship between the Transformational leadership style and leader effectiveness among middle managers within an organization?

2. What is the relationship between Transactional leadership style and leader effectiveness among middle managers within an organization?

3. What is the relationship between Passive leadership style and leader effectiveness among middle managers within an organization?

4. What are the primary competencies for effective healthcare managers?

5. Which leadership style has been found to have the most significant impact on leader effectiveness and efficiencies?

\section{Literature Review}

The literature review for this study provides an examination of the multiple approaches to leadership styles and their outcomes of leadership in healthcare organizations. This literature review includes theories of leadership and findings present potential leadership methods associated with mid-level managers. This qualitative study proposal addresses mid-level managers effectiveness in regard of transactional, transformation, and passive leadership styles in middle level management in healthcare organizations.

\section{A. Mid-level Leadership}

The healthcare system has been theorized as a product of personal health services and this requires managers to assure safety, efficiency, and qualities of service to meet the needs of the patient (Shortell \& Kalunzy 2006). Furthermore, these realities present a complex and challenging management environment (Shortell \& Kalunzy 2006). The need for mid-level managers is increasing in today's healthcare environment as mid-level managers function as the conduit of influence between upper management and the front-line employees and are vital for implementing and sustaining quality practices to meet healthcare complexities (Chilgren 2008). Middle managers are defined as staff with a supervisory capacity other than senior leaders and are noted as department managers, program managers, nurse managers, administrative directors, and frontline supervisors (Engle et al. 2017).

Engle et al (2017) did a substantial study on the role of middle managers in hospital settings. The purpose of the study conducted by Engle et al (2017) was to increase the understanding of the influence of middle managers in organizations by analyzing qualitative data from 17 Veterans Affairs Medical Centers with high and low potential to change organizational practices. Engle et al (2017) examined 98 consultations with employees ranging from senior leaders to frontline staff members to identify themes within an a priori framework that reflect middle manager undertakings. Middle managers are known to play key roles in hospital settings because they bridge gaps between senior leaders and frontline staff, but little research has focused on middle managers roles in implementing new practices. Middle managers play important roles in health care organizations by facilitating the implementation of innovative and quality improvement initiatives and current literature has confirmed that individual and organizational factors influence quality improvement effectiveness (Engle et al. 2017). This emphasis has been on senior leaders but lacks focus on the influence of middle managers on organizational change efforts. Therefore, there is a need for further research to measure or estimate middle level management effectiveness in healthcare settings (Engle et al. 2017). Middle managers can facilitate or enhance innovation implementation processes, implement change, and improve organizational performance. Middle managers are situated between senior leaders and frontline staff of an organization and can either bridge or widen information gaps that may influence innovation implementation in positive or negative ways (Engle et al. 2017).

Engle et al. (2017) further expand and theorize the role of middle or mid-level managers in a healthcare organization expressed by their commitment to innovation implementation in the following four different ways: (a) mid-level managers diffuse information to give employees the necessary information in regard to innovation implementation, (b) mid-level managers synthesize information to provide appropriate examples to support employees understanding in how innovations are implemented in 
the workplace, (c) mid-level managers serve as mediators between strategy and day-to-day undertakings to give staff the tools required to implement innovations, and (d) mid-level managers sell innovation implementation execution processes to encourage staff members to use it consistently and effectively (Engle et al. 2017).

The Organizational Transformation Model developed by Lukas et al (2007) can be used as a basis for further examining the behaviors of middle managers and their contributions to facilitating transformations within an organization. The Organizational Transformation Model demonstrates that improvement in health care organizations was more impactful when middle managers were committed to quality and actively involved in the redesign process being fully aligned around the importance of quality improvement (Lukas et al. 2007).

Middle managers play a key role in overcoming the challenges of innovation implementation as has been offered by many studies in the field (Shortell \& Kalunzy 2006; Chilgren 2008; Engle, et al. 2017). A study conducted by Birken et al (2015) focused on how middle managers oversee the implementation of an innovation project to reduce health disparities. They found that middle managers improved the effectiveness of Chronic Care Model implementation in their healthcare organization (Birken, et al. 2015). It is theorized that middle managers can exploit their unique position that falls between upper and lower levels in the healthcare organization by engaging in ambidextrous learning that is critical to implementing and sustaining fundamental change (March 1991). This organizational learning perspective offers an innovative way of framing the role of mid-level managers through explorative and exploitative activities that additionally reflects the necessary organizational framework in which they manage (Floyd \& Wooldridge 1997). The theories of both implementation and change management support the importance of leadership at all levels (Floyd \& Wooldrige 1997). In a follow up article, Wooldridge, Schmid \& Floyd (2008) proposed that leadership at the middle level of management consists of those individuals who have supervisory or managerial roles that are neither front-line staff nor senior leadership team members and therefore can facilitate radical organizational change more efficiently.

The increasing knowledge about the vital role that middle management plays in strategic change in all sectors, but particularly the healthcare sector, should result in a higher value placed on middle managers in these industries. However, it appears that there is a very limited understanding of what contributions middle managers make towards strategy and organizational change because despite these consistent results, the middle management is often not involved to the extend it should be when strategic changes occur (Gutberg \& Berta 2017). Significant literature and theories of leadership focus upon the role of senior leadership while ignoring or minimizing the role of the middle management. Gutberg \& Berta (2017) found that the potential for middle managers to contribute to strategic organizational activities, a relatively recent consideration in the healthcare industry could substantially change the landscape of the industry by improving efficiencies and reducing costs. Literature demonstrates that middle managers have the ability to facilitate communication throughout and within an organization (Birken, Lee \& Weiner 2012). Middle managers not only leverage but exploit their access to knowledge and networks and serve as a conduit to transfer information regarding the implementation strategy down to the lower levels of the organization, while simultaneously translating the strategy or vision into executable processes and steps to be adopted by front-line employees (Birken, Lee \& Weiner 2012). Middle managers accomplish this by directing the flow of information both upward and downward by communicating from the front-line staff back up to the senior leadership level.

A recent study by Birken, et al. (2016) found that middle managers ranked the highest in diffusing and synthesizing information and stated that future studies are required to assess the relationship between hypothesized roles and the effectiveness with which innovations are implemented in practice. It was also noted that middle managers have received increased attention in recent healthcare innovation implementation research and this emerging literature suggests that middle managers influence innovation implementation by bridging informational gaps between top managers and frontline employees that might otherwise impede innovation implementation in healthcare settings (Birken, et al, 2016). 


\section{B. Leadership Styles}

The role of middle managers in terms of the strategic benefits has been discussed in the first part of this section. However, now we need to try to understand what style of leadership is the most effective at the mid-management level. The Full Range Leadership Model encompasses transformational, transactional, and passive styles and is regarded as the leading model for describing leadership styles (Nash, Davies \& Moore, 2017). The Full Range Leadership Model is comprised of three different leadership styles: transformational, transaction, and passive as shown in Figure 1.

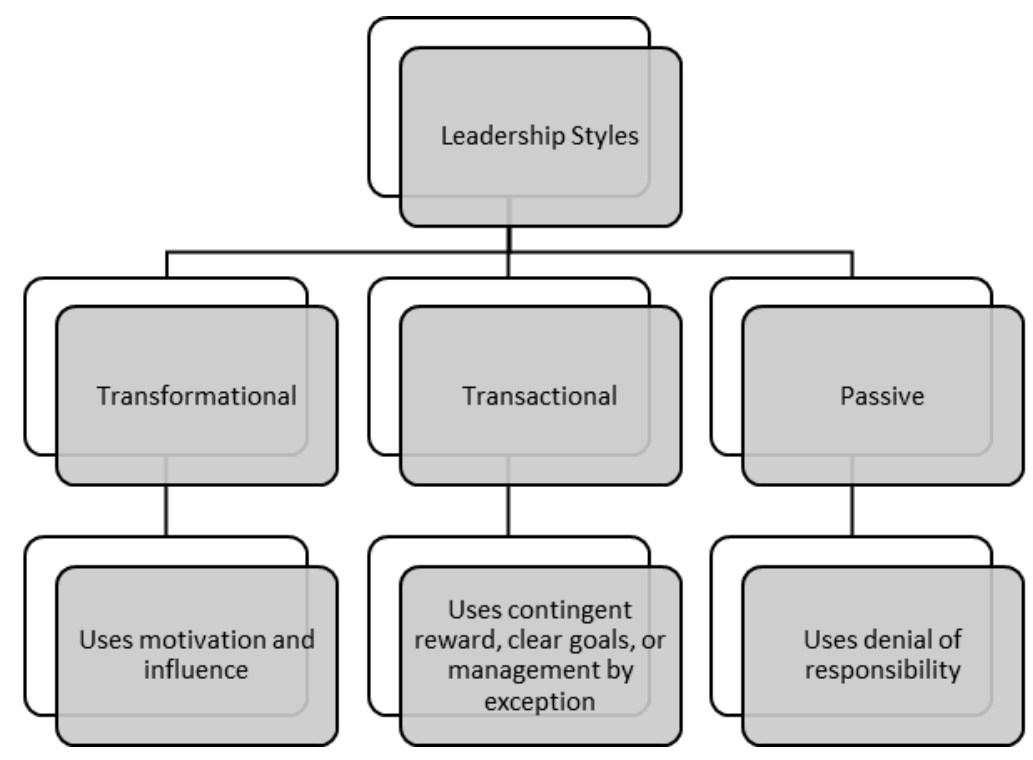

Figure 1. Types of Leadership Styles based on Full Range Leadership Model

Tice (2007) explores the impact of leadership styles and traits that influence styles associated with middle managers in healthcare, but further research is needed to identify which leadership style is most effective for mid-level managers in healthcare settings. Research shows that middle managers should be flexible and adaptable when it comes to managing their behavior and actions (Northouse 2007). This is an important consideration in regard of leadership style as Bass (2008) states the requirements for effective leadership differs based upon the specific role of the leader and the functions they perform within the organization. Ultimately, the performance of healthcare organizations is impacted by the extent to which middle managers have a positive effect on employee performance (Olivo 2007).

Hana \& Kirhaug (2014) confirmed that leadership in rural general practice healthcare settings can be identified in terms of change, task, and relation styles and that change style is perceived most by staff. Leadership in general practice healthcare settings may differ from leadership in traditional organizations and that task style may also include some interpersonal aspects (Hana \& Kirhaug, 2014). Support staff in healthcare represent stability and hence influence culture, which makes it essential to identify whether they are influencing change due to their innate leadership style or the actual need for leadership among employees in this industry (Hana \& Kirkhaug 2014).

The Full Range Leadership Model suggests that a specific set of leadership components is necessary for effective leadership these component parameters include a large portion of transformational leadership execution, larger levels of transactional leadership usage, and a minimum level of passive or laissez-faire leadership (Saravo, Netzel \& Kiesewetter 2017). In research fields that are typically concerned with leadership studies, the Full Range Leadership Model is the most comprehensive and most researched leadership model and aims to describe the full range of leadership behaviors. This includes aspects from the suggested desired active leadership perspective that is called transformation leadership to the undesired passive leadership perspective that is called laissez-faire leadership (Saravo, Netzel \& Kiesewetter 2017).

1) Transformational Leadership 
Bass (2008) defines a transformational leader as an individual who motivates his or her followers to exceed their previous expectations. Leaders with an appealing vision for their team employ transformational leadership and this refers to those leaders that intellectually stimulate staff in a way that is demanding and appreciative of the individual needs of the staff members (Saravo, Netzel \& Kiesewetter, 2017). Spinelli (2006) noted that leader effectiveness, particularly in the hospital administrative environment, was one of the first areas where research on transformational leadership styles was performed. Transformational leadership consists of managers who act as role models and are able to prepare and verbalize an inspiring vision for the workplace's future (Saravo, Netzel \& Kiesewetter, 2017). Transformational leaders encourage employees to be creative and innovative, empower subordinates to autonomously make their own decisions, and employ coaching to ensure that subordinates are able to develop their capabilities (Saravo, Netzel \& Kiesewetter 2017). Research provided by multiple metaanalyses and reviews have showcased the positive effects of transformational leadership in regard of workplace productivity, employee effectiveness and job satisfaction, and overall organizational performance (Saravo, Netzel \& Kiesewetter 2017). Within healthcare organizations, Transformational leadership has been found to be associated with better patient outcomes and has been identified as specifically efficient for change processes (Saravo, Netzel \& Kiesewetter 2017). Transformational leadership motivates workers through inspiring them to rise above their personal interest for the good of the organization and its goals (Aberese-Ako, et al, 2018) because it inspires creativity, flexibility, and appeals to the inner motivation of employees (Hartviksen, et al. 2018). Thus, transformational leadership is suitable for transforming individuals and the entire organization, which requires learning to facilitate adoption, transformation of organizational culture, and progress (Aberese-Ako, et al. 2018). It is important to note that while transformational leadership might be enough to facilitate change, it may not always be sufficient to adapt employees to change in the most efficient manner (Saravo, Netzel \& Kiesewetter 2017).

\section{2) Transactional Leadership}

Aberese-Ako, et al. (2018) defines transactional leadership as leadership style that ensures that workers operate in accordance to the rules and regulations of the organization, punishes those who do not work hard, and rewards those who are hard working. The transactional leadership style is a form of carrot-stick approach to leadership and includes leaders who would wait until things go wrong, and then they come in to punish, and lastly correct and teach (Aberese-Ako, et al. 2018).As specified by the Full Range Leadership Model, transactional leadership is a rather active leadership style, and due to the different natures of its sub-constructs has been associated with mixed results (Saravo, Netzel \& Kiesewetter, 2017). Transactional leaders are known to apply influence on subordinates by exchanging benefits for exceptional performance and provide incentives to the staff when they have achieved defined goals (Saravo, Netzel \& Kiesewetter 2017). Transactional leadership relates to external motivation, contingent reinforcement, guidelines, command, and control (Hartviksen, et al, 2018). Due to these sub-constructs, contingent reward has been noted to be effective, whereas the management by exception sub-construct is typically not considered to be an effective leadership style (Saravo, Netzel \& Kiesewetter 2017). From research posited from organizational change, the transactional leadership sub-construct of contingent reward is vital for the effective management of change processes; noted particularly in regard to managers being specific, providing feedback, and evaluating the change process (Saravo, Netzel \& Kiesewetter 2017).

It has been noted that transactional leadership is more feasible to organizations in modern times by getting their staff to perform tasks for a fee and has proven to be effective for change and efficiency compared to other leadership styles (Aberese-Ako, et al, 2018). However, through quantitative analysis, a research study revealed that sickness rates of $5.7 \%$ that were slightly higher than the $5.2 \%$ average national sickness rate in The Netherlands in 2010 were associated with a general pattern of low employee satisfaction and transactional leadership style (Elshout, Scherp \& van der Feltz-Cornelis 2013). This association, in contrast to transformational leadership style, was described best by: (1) communication between the manager and employees; (2) the application of sickness protocols by the managers; and (3) leadership style of the manager (Elshout, Scherp \& van der Feltz-Cornelis 2013).

Aarons (2006) stated that transactional leadership is based more on "exchanges" between the leader and follower, in which followers are rewarded for meeting specific goals and that transactional leadership is more practical in nature because of its emphasis on meeting specific targets or objectives. 
The transactional leadership has been noted to motivate workers through an exchange process involving extrinsic rewards, rules, and compliance (Aberese-Ako, et al. 2018). It has been discovered that the subordinates of transactional leaders, in contrast to those who have transformational leaders, are not necessarily expected to think innovatively and may be monitored on the basis of predetermined criteria (Aarons 2006). Hartviksen, et al. (2018) confirmed that transactional leadership style currently dominates in the healthcare industry, and this is an important consideration when calculating the current avoidable costs associated with the industry. Healthcare management has been traditionally characterized by strategic planning and implementing concrete tasks in a transactional leadership structure that is based on hierarchical and linear leadership styles (Hartviksen, et al. 2018).

Basran, et al (2019) suggests that transactional leadership at its simplest seems like an antisocial leadership style and uses basically a threat and self-focused competitive orientation to challenge employees to compete for resources and to finds status and social position in the world. Transactional leaders often utilize the language of threat to generate support or subdue dissent and are insensitive to the harm they may cause, and history shows they are often power-orientated and have very destructive impacts on the organizations they lead (Basran, et al. 2019).

\section{3) Passive Leadership}

Unlike the transformational and transactional leadership styles, the passive leadership style is found in a leader that does not take charge of their leadership role and is the least active form of leadership (Saravo, Netzel \& Kiesewetter 2017). Laissez-faire or the passive leadership style is viewed as leadership that does not make decisions to guide, correct or inspire subordinates to do their work and chooses not to apply the rules and allows workers to do whatever they choose, even when they do not work in the interest of the organization (Aberese-Ako, et al. 2018). The laissez-faire leadership style involves a leader who does not make decisions and research shows that this results in rarely observed changes and has a negative impact on the culture in healthcare organizations (Sfantou, et al. 2017). Barling \& Frone (2017) conducted a quantitative study that found that passive leadership is directly related to a poor work environment and is directly and/or indirectly related to several types of employee harm, higher levels of psychological work fatigue, poorer mental health and overall work attitude. Dóci, Stouten \& Hofmans (2015), stated in accordance with the Full Range of Leadership Model that the passive end of the leadership spectrum lies the lack of leadership and that Laissez faire, a form of passive leadership style, is characterized by the avoidance, even in dire circumstances, of taking leadership responsibilities, decisions, and actions.

\section{Ideal Style of Leadership in the Healthcare Industry}

The literature analysis shows that among all the styles of leadership, the transformational leadership style is best when utilized for attaining employee satisfaction and there is strong difference in leadership styles when comparing the worst and best performing organizations in terms of employee satisfaction and absenteeism. Elshout, Scherp \& van der Feltz-Cornelis (2013) found that the worst departments in healthcare organizations tend to have a manager that employs a transactional style of leadership whereas the best departments tend to have managers that employed a transformational style of leadership. Multiple studies have shown that leadership is necessary for improved performance and innovation implementation in a healthcare organization (Engle, et al. 2017). Additional research is needed to evaluate how mid-level managers influence implementation effectiveness as they facilitate or enhance innovation implementation procedures and improve healthcare organizational performance (Engle, et al. 2017). Mid-level managers are positioned between senior leaders and frontline staff of an organization and this enables them to bridge or create information gaps that may influence innovation implementation (Engle, et al. 2017). Therefore, additional research is needed to expand the understanding of how differences in the actions of mid-level managers based on their leadership style influence improvement efforts in healthcare organizations.

\section{Proposed Quantitative Model and Methodology}

The quantitative analysis of the shortfall in leadership competencies of healthcare mid-level managers resulting in inefficient and poor-quality health care (North 2008) can be conducted using the outcomes from the literature. This quantitative non-experimental correlational study would examine the statistical relationships between the independent variables of three leadership styles (transformational, transactional, 
and passive leadership) and the dependent variable of leadership effectiveness among mid-level managers in the healthcare industry. The analysis would use the concepts of clearly defined independent variables transformational leadership, transactional leadership, passive leadership, and leader effectiveness by quantifying them. A correlational design combined with multiple linear regression analysis can be used for estimating the relationships between the categorical dependent variable leadership effectiveness and each of the independent variables (transformational leadership, transactional leadership, and passive leadership) to examine the relationships to leadership effectiveness.

The target population for this research study would be mid-level managers in the healthcare industry from three groups: nursing managers, ancillary clinical managers, and other healthcare management staff. The participants can be drawn from any healthcare organization. Data for this study can be collected by distributing the Multifactor Leadership Questionnaire (Bass 2008), which is readily available. The Multifactor Leadership Questionnaire is a tool developed to measure leadership styles and effectiveness to assess a manager's ability to interpret and understand their leadership impact (Bass 2008). The Multifactor Leadership Questionnaire will be used to collect data on the three primary independent variables: transformational leadership, transactional leadership, and passive leadership as well as the dependent variable, leader effectiveness.

\section{A. Survey Variables}

a. Leader effectiveness - for this dependent variable, the Multifactor Leadership Questionnaire includes about nine items where each item is rated on a Likert scale ranging from 0 (not at all) to 4 (frequently, if not always). These nine items will be added together to provide an overall score ranging between 0 and 36, with higher scores representing a higher level of effectiveness of the individual as a leader.

b. Transformational leadership - the Multifactor Leadership Questionnaire includes about 20 items where each item is rated on a Likert scale ranging from 0 (not at all) to 4 (frequently, if not always). These 20 items can be added together to provide an overall score ranging between 0 and 80 , with higher scores representing a tendency toward the use of transformational leadership methods.

c. Transactional leadership - the Multifactor Leadership Questionnaire includes about 12 items where each item is rated on a Likert scale ranging from 0 (not at all) to 4 (frequently, if not always). These 12 items will be added together to provide an overall score ranging between 0 and 48, with higher scores representing a tendency toward the use of transactional leadership methods.

d. Passive leadership - the Multifactor Leadership Questionnaire includes about 4 items where each item is rated on a Likert scale ranging from 0 (not at all) to 4 (frequently, if not always). These 4 items will be added together to provide an overall score ranging between 0 and 16, with higher scores representing a tendency toward the use of passive leadership methods.

e. Demographic variables - gender, age, highest educational attainment, and number of years of experience in the current role of the participants will function as control variables in the survey. All efforts should be made to exclude any personally identifiable information in order to protect participants in the study.

\section{B. Model}

The proposed quantitative analysis aims to identify whether the style of leadership that is primarily followed by an individual has an impact on his/her effectiveness as a leader. This can be mathematically presented as follows:

\section{$L E=f($ Transform, Transact, Passive, gender, age, education, experience $)$}

One would expect that an individual demonstrates multiple facets of the various leadership styles. However, there is likely to be one style that dominates over the other two for each individual. This type of model, if estimated using a regression analysis approach can help identify not only if the dominant style impacts the effectiveness of the leader but also can help identify how much more (or less) effective a leader can be by increasing or reducing the reliance on the other types of styles based upon the items they answered in the questionnaire. This can have long standing implications on helping individuals become 
more effective leaders in the context of their own strengths and weaknesses by recognizing the potential for improvement. Thus, while the survey itself would be anonymous, an individual who sees the results of this analysis can easily identify which questions pertaining to which leadership style can help them improve their own scores, in the context of their own specific roles in their workplace.

\section{Data collection Method}

According to Cohen (1988), the level of desired power in social sciences is 0.80 ; therefore, this type of data collection would require at least 77 participants based on the statistical power analysis. A researcher exploring this type of modeling would need to send invitations for participation in the survey to at least 200 healthcare mid-level managers to ensure an excellent rate of response. The survey will be open for a 7-week period for completion and each participant will complete the Multifactor Leadership Questionnaire. Instructions will be provided in an electronic format to all participants review with the initial solicitation for participants. The data collected from SurveyMonkey can be imported into a statistical software for analysis. Basic descriptive and inferential statistical analysis can be performed on all the variables with particular attention paid to the Leader Effectiveness scale (the dependent variable). Since this type of data collection would require prior IRB approval, it would be done at a later stage (beyond the scope of the current paper).

\section{Conclusion and Future Scope}

The aim of this study was to explore how mid-level managers impact healthcare organizations by looking at their leadership style through the lenses of different leadership conceptual frameworks and methodologies. Based upon the literature analysis, we propose a potential quantitative model that can allow mid-level managers as well as the industry in general to identify which leadership styles would result in the highest levels of effectiveness in their firm. This type of quantitative project can contribute to implementation science as well by examining implementation leadership and has the potential to make significant contributions towards the research and practice of leadership skills and styles. While this paper is the first step in conveying the model, it incorporates an understanding of various leadership styles that can increase the success rate for mid-level managers in the healthcare industry by reducing avoidable costs and improving the efficiency of workers in healthcare by providing them with the power to change the structure for the better.

\section{References}

Aarons, G. A. 2006. "Transformational and Transactional Leadership: Association with Attitudes Toward Evidence-Based Practice”, Psychiatric Services (Washington, D.C.), 57(8): 1162-1169. http://doi.org/10.1176/appi.ps.57.8.1162.

Bass, B. M. 2008. The Bass Handbook of Leadership (4th ed.). New York, NY: Free Press.

Aberese-Ako, M., Agyepong, I. A., \& van Dijk, H. 2018. "Leadership styles in two Ghanaian hospitals in a challenging environment", Health Policy and Planning, 33(suppl_2), ii16-ii26. doi:10.1093/heapol/czy038.

Aitken, M. 2013. "Avoidable Costs in U.S. Healthcare." IMS Institute for Healthcare Informatics. Retrieved from: http://offers.premierinc.com/rs/381-NBB-525/images/Avoidable_Costs_in\%20_US_HealthcareIHII_AvoidableCosts_2013\%5B1\%5D.pdf.

Barling, J., \& Frone, M. R. 2017. "If Only my Leader Would just Do Something! Passive Leadership Undermines Employee Well-being Through Role Stressors and Psychological Resource Depletion." Stress and Health: Journal of the International Society for the Investigation of Stress 33(3): 211-222. doi:10.1002/smi.2697.

Basran, J., Pires, C., Matos, M., McEwan, K., \& Gilbert, P. 2019. "Styles of Leadership, Fears of Compassion, and Competing to Avoid Inferiority." Frontiers in Psychology 9: 2460. doi:10.3389/fpsyg.2018.02460.

Birken S, Lee S-Y, Weiner B. 2012. "Uncovering middle managers' role in healthcare innovation implementation." Implement Science: IS 7(1): 28. doi: 10.1186/1748-5908-7-28.

Birken, S. A., Lee, S. Y., Weiner, B. J., Chin, M. H., Chiu, M., \& Schaefer, C. T. 2015. "From strategy to action: how top managers' support increases middle managers' commitment to innovation implementation in health care organizations." Health care Management Review 40(2): 159-168. doi:10.1097/HMR.0000000000000018.

Birken, S. A., DiMartino, L. D., Kirk, M. A., Lee, S. Y., McClelland, M., \& Albert, N. M. 2016. "Elaborating on theory with middle managers' experience implementing healthcare innovations in practice.” Implementation Science: IS, 11, 2. doi:10.1186/s13012-015-0362-6.

Chilgren, A. A. 2008. "Managers and the new definition of quality.” Journal of Healthcare Management 53: $221-229$.

Cohen, J. 1988. Statistical Power Analysis for the Social Sciences (2nd ed.). New York, NY: Routledge Academic. 
Dóci, E., Stouten, J., \& Hofmans, J. 2015. "The cognitive-behavioral system of leadership: cognitive antecedents of active and passive leadership behaviors." Frontiers in Psychology 6: 1344. doi:10.3389/fpsyg.2015.01344.

Elshout, R., Scherp, E., \& van der Feltz-Cornelis, C. M. 2013. "Understanding the link between leadership style, employee satisfaction, and absenteeism: a mixed methods design study in a mental health care institution." Neuropsychiatric Disease and Treatment 9: 823-837. doi:10.2147/NDT.S43755.

Engle, R., Lopez, E., Gormley, K., Chan, J., Charns, M. \& Lukas, C. 2017. "What roles do middle managers play in implementation of innovative practices?" Health Care Management Review 42 (1): 14-27. doi: 10.1097/HMR.0000000000000090.

Floyd SW, Wooldridge B. 1997. "Middle management's strategic influence and organizational performance." Journal of Management Studies 34(3): 465-485. doi: 10.1111/1467-6486.00059.

Gutberg, J., \& Berta, W. 2017. "Understanding middle managers' influence in implementing patient safety culture.” BMC Health Services Research 17(1): 582. doi:10.1186/s12913-017-2533-4.

Hana, J., \& Kirkhaug, R. 2014. "Physicians' leadership styles in rural primary medical care: how are they perceived by staff?" Scandinavian Journal of Primary Health Care 32(1): 4-10. doi:10.3109/02813432.2013.874083.

Hartviksen, T. A., Sjolie, B. M., Aspfors, J., \& Uhrenfeldt, L. 2018. "Healthcare middle managers` experiences developing leadership capacity and capability in a public funded learning network." BMC Health Services Research 18(1): 433. doi:10.1186/s12913-018-3259-7.

Lantz, P. M., \& Maryland, P. A. 2008. "Gender and leadership in healthcare administration: 21 st century progress and challenges." Journal of Healthcare Management 53: 291-303.

Lukas C. V., Engle R. L., Holmes S. K., Parker V. A., Petzel R. A., Seibert M. N., Sullivan J. L. 2010. "Strengthening organizations to implement evidence-based clinical practices." Health Care Management Review 35(3): $235-245$.

March JG. 1991. "Exploration and exploitation in organizational learning." Organizational Science 2(1):71-87. doi: 10.1287/orsc.2.1.71.

Nash, M., Davies, A., \& Moore, R. 2017. "What style of leadership do women in STEM fields perform? Findings from an international survey." PloS One, 12(10), e0185727. doi:10.1371/journal.pone.0185727.

North, M. 2008. "Managing people when you really don't like to." Healthcare Financial Management 62: 110-112.

Northouse, P. G. 2007. Leadership Theory and Practice (4th ed.). Thousand Oaks, CA: Sage.

Olivo, T. 2007. "The connective link between organizational culture and performance in healthcare organizations." Healthcare Performance Solutions, Retrieved from: http:/www.healthcareps.com.

Pelote, V. \& Route, L. 2007. Masterpieces in Healthcare Leadership. Sudbury, MA: Jones and Bartlett.

Saravo, B., Netzel, J., \& Kiesewetter, J. 2017. "The need for strong clinical leaders - Transformational and transactional leadership as a framework for resident leadership training." PloS One, 12(8), e0183019. doi:10.1371/journal.pone.0183019.

Sfantou, D. F., Laliotis, A., Patelarou, A. E., Sifaki- Pistolla, D., Matalliotakis, M., \& Patelarou, E. 2017. "Importance of Leadership Style towards Quality of Care Measures in Healthcare Settings: A Systematic Review." Healthcare 5(4): 73. http://doi.org/10.3390/healthcare5040073.

Shortell, S. M., \& Kaluzny, A.D. 2006. Healthcare Management: A Text in Organizational Theory and Behavior. New York, NY: John Wiley and Sons.

Spinelli, R. J. 2006. "The applicability of Bass's model of transformational, transactional, and laissez-faire leadership in the hospital administrative environment." Hospital Topics 3: 69-80.

Tice, C. 2007. "Building the 21st century leader." Entrepreneur 2: 64-69.

Wooldridge B, Schmid T, Floyd SW. 2008. "The middle management perspective on strategy process: contributions, synthesis, and future research." Journal of Management 34(6):1190-1221.

Wurster, C. J. 2009. "Strategic, political, and cultural aspects of IT implementation: improving the efficacy of an IT system in a large hospital." Journal of Healthcare Management 54: 191-201. 